\title{
Asymptotic methods for solving boundary value eigenvalue problems
}

\author{
Galina Zhukova ${ }^{1, *}$ \\ ${ }^{1}$ Financial University under the Government of the Russian Federation, 125993, Leningradskiy \\ Avenue, 49, Moscow, Russian Federation
}

\begin{abstract}
The aim of the study is an approximate construction with a given accuracy of solutions of boundary value problems for eigenvalues under various types of boundary conditions. It is shown that the problem of finding approximate large eigenvalues of boundary value problems is reduced to the analysis and solution of singularly perturbed differential equations with variable coefficients. Methods used: asymptotic diagram method developed to construct the asymptotic behavior of solutions of singularly perturbed differential equations and systems; methods of numerical integration of boundary value problems. The main results obtained are: the asymptotics of the required accuracy are constructed in the analytical form for the eigenvalues and eigenfunctions of the boundary value problems under various boundary conditions; analysis of the computational capabilities of the practical use of the constructed asymptotics in comparison with the results of numerical integration.
\end{abstract}

\section{Introduction}

The need for an approximate solution of boundary value problems often arises in the study of a wide variety of applied problems. The processes of heat and mass transfer can be described on the basis of differential equations of continuum mechanics, which reflect the objective laws of conservation of mass, momentum and energy. The creation and solution of adequate mathematical models of these processes, where boundary value problems for eigenvalues for differential equations often arise, allows to reduce the amount of experimental research that needs to be carried out, for example, to achieve the required parameters of heat exchangers and systems.

In order to increase the overall efficiency of systems, for example, the conversion of solar energy into thermal energy, studies have been carried out in recent decades, the authors of which propose using liquid fusible metals as heat transfer liquid in such systems [1-2], and come to consider differential equations with boundary conditions.

For specifics, we also indicate that heat transfer in a flat channel with a fully developed turbulent flow [3] is described by the boundary value problem (1):

*Corresponding author : galsevzhukova@mail.ru 


$$
\left\{\begin{array}{l}
x^{\prime \prime}+\lambda^{2}\left(1-t^{2}\right) f(t) x=0 \\
x(0)=x(1)=0
\end{array}\right.
$$

Here $f(t)>0$ at $t \in[0 ; 1], \lambda$ is a large parameter. Another example is the natural vibrations of an absolutely flexible, heavy, inextensible, uniformly loaded rope of length $l_{0}$ suspended at one end, which satisfy the following boundary value problem (2):

$$
\left\{\begin{array}{l}
\varepsilon^{2}\left(\left(G+p g F\left(l_{0}-x\right)\right) y^{\prime}\right)^{\prime}+p F y=0 \\
y(0)=0, \quad y^{\prime \prime}\left(l_{0}\right)=0
\end{array}\right.
$$

( $g$ is the acceleration of gravity; $G$ is the weight of the load to be lifted; $F$ is the crosssectional area of the rope; $P$ is the density; $\omega$ is the circular frequency of movement of the rope particles; the number $\varepsilon=1 / \omega$ is a small parameter of the problem) [4].

Consider the problem of approximate finding large eigenvalues $\lambda$ of a boundary value problem, given in general form:

$$
\begin{gathered}
f_{2}(t) x^{\prime \prime}+f_{1}(t) x^{\prime}+f_{0}(t) x+\lambda^{2} f_{3}(t) x=0, \\
\left\{\begin{array}{l}
A_{1} x(0)+B_{1} x^{\prime}(0)=0, \\
A_{2} x(T)+B_{2} x^{\prime}(T)=0,
\end{array}\right.
\end{gathered}
$$

where $f_{j}(t), j=\overline{0 ; 3}$ are given sufficiently smooth for $t \in[0 ; T]$ functions; $A_{j}, B_{j}(j=\overline{1 ; 2})$ are given constant numbers, some of which may, in particular, be zero.

Recall that the parameter values $\lambda$ at which problem (3) - (4) has nontrivial solutions on $[0 ; T]$ are called eigenvalues, and the corresponding solutions are called eigenfunctions of the boundary value problem. The eigenvalue problems include not only the above examples, but also the problems of determining the natural vibrations of material systems with distributed characteristics: transverse and longitudinal vibrations of a string, longitudinal vibrations of an elastic rod, sound vibrations in pipes, electrical vibrations in wires, etc. Some boundary value problems, due to their great practical importance in mechanics, physics, and technology, have been studied by many authors who have used various approaches for this, in particular, asymptotic methods [5-11].

In the work, problem (3) - (4) reduces to an eigenvalue problem for a singularly perturbed differential equation. For its analysis and approximate determination of eigenvalues and eigenfunctions, it is proposed to use the diagram method developed by the author [12-14] to construct the asymptotic behavior of the solutions of singularly perturbed differential equations and systems of any order. As a result of this approach, the asymptotics of the eigenvalues and eigenfunctions of the boundary value problems under various types of boundary conditions are constructed in an analytical form. By analogy with [15-16], a comparison of the computational efficiency of the practical use of the results is made. 


\section{Research method}

Let functions $f_{j}(t), j=\overline{0 ; 3}$ in equation (3) satisfy the conditions:

$$
f_{2}(t) \neq 0, \quad f_{3}(t) \neq 0, \quad \frac{f_{3}(t)}{f_{2}(t)}>0 \quad(t \in[0 ; T]) .
$$

Since the statement of the problem $\lambda$ are large eigenvalues of the boundary value problem (3) - (4), we introduce a small parameter by the formula:

$$
\varepsilon=\frac{1}{\lambda}
$$

Then equation (3) takes the form

$$
\varepsilon^{2} f_{2}(t) x^{\prime \prime}+\varepsilon^{2} f_{1}(t) x^{\prime}+\left(f_{3}(t)+\varepsilon^{2} f_{0}(t)\right) x=0,
$$

and it is a scalar linear singularly perturbed second-order differential equation with variable coefficients.

Note that (7) is a special case of a singularly perturbed differential equation of the form

$$
\sum_{v=0}^{n} \varepsilon^{p_{v}} a_{v}(t ; \varepsilon) x^{(v)}=0
$$

where

$$
a_{0}(t ; \varepsilon) \not 0, a_{n}(t ; \varepsilon) \neq 0 \quad\left(t \in[0 ; T], \varepsilon \in\left[0 ; \varepsilon_{0}\right]\right) ;
$$

functions $a_{v}(t ; \varepsilon)$ are real-valued, have uniform asymptotic expansions at $[0 ; T]$

$$
a_{v}(t ; \varepsilon) \sim \sum_{s=0}^{\infty} \varepsilon^{s} a_{v s}(t), \quad \varepsilon \rightarrow+0,
$$

where $a_{v s} \in C^{\infty}[0 ; T] ; p_{0}, \ldots, p_{n}-$ non-negative constant numbers, $p_{n}>0$. In the case under consideration, for equation (7) we have:

$$
\begin{gathered}
n=2 ; \quad p_{0}=0 ; \quad p_{2}=p_{1}=2 ; \\
a_{20}(t)=f_{2}(t) ; \quad a_{10}(t)=f_{1}(t) ; \quad a_{00}(t)=f_{3}(t) ; \\
a_{01}(t)=0 ; \quad a_{02}(t)=f_{0}(t) ; \quad a_{v s}(t) \equiv 0 \quad(v=1,2 ; s \geq 1) ; \quad a_{0 s}(t) \equiv 0 \quad(s \geq 3) .
\end{gathered}
$$

To construct an approximate solution of a singularly perturbed differential equation (7), we apply the asymptotic method of diagrams proposed in [12-14]. Recall [12] that for a 
singularly perturbed differential equation (8), the first diagram is a convex polygonal line constructed using Newton's geometric technique from a set of points

$$
C_{0}=\left(0 ; \rho_{0}\right), \ldots, C_{l}=\left(l ; \rho_{l}\right), \ldots, C_{n}=\left(n ; \rho_{n}\right),
$$

where

$$
\rho_{n}=p_{n}, \quad \rho_{l}=\min _{v=l, n} p_{v} \quad(l=\overline{1, n-1}), \quad \rho_{0}=p_{0}
$$

The index $l$ takes those of values $0, \ldots, n-1$ at which $F_{l 0}[z] \not \equiv 0$, where the functions $F_{l s}[z]$ are calculated through the coefficients of equation (8) by the formulas:

$$
\left\{\begin{aligned}
& F_{0 s}[z] \equiv a_{0 s}(t), \quad F_{n s}[z] \equiv a_{n s}(t) z^{n}, \\
& F_{l s}[z] \equiv a_{l, s-p_{l}+\rho_{l}}(t) z^{l}+\sum_{v=l+1}^{n} a_{v, s-p_{v}+\rho_{l}}(t) \sum_{i_{1}=l-1}^{v-1} \sum_{i_{2}=l-2}^{i_{1}-1} \ldots \\
& \ldots \sum_{i_{l-1}=1}^{i_{l-2}-1} r_{v l} \prod_{c=0}^{l-1}\left(z^{\left(i_{c}-1-i_{c+1}\right)}\right), i=\overline{1, n-1 .}
\end{aligned}\right.
$$

In the case of a singularly perturbed second-order differential equation of the form (7), the indicated formulas (9)-(16) are significantly simplified. First, then only three points are involved in the construction of the first diagram of equation $(7)$ : $(0 ; 0),(1 ; 2)$ and $(2 ; 2)$. Therefore, based on Newton's geometric technique, the first diagram of differential equation (7) contains only one link with the beginning at the point $(0 ; 0)$ and the ending point $(2 ; 2)$. Point $(1 ; 2)$ at any values of the coefficients of equation $(7)$ does not fall on the diagram (located strictly above it).

The projection length of the diagram on the abscissa axis is equal to the number 2 , that is, it is equal to the order of the differential equation (7).

The slope coefficient of a chart link is equal to a number 1 . The defining equation of the link of the diagram has the form:

$$
f_{2}(t) \cdot\left(\mu_{0}\right)^{2}+f_{3}(t)=0 .
$$

Therefore, under conditions (5), the defining equation (17) of the only link in the first diagram of differential equation (7) has two simple complex conjugate roots:

$$
\left(\mu_{0}\right)_{1,2}(t)= \pm \mathrm{i} \sqrt{f_{3}(t) / f_{2}(t)}
$$

$\mathrm{i}$ is the imaginary unit.

Therefore, based on the properties of the diagram method, we conclude: when conditions (5) are fulfilled, approximations of any order $\mathrm{O}\left(\varepsilon^{m}\right)$ to the fundamental solutions of differential equation (7) will be constructed by the formula: 


$$
\exp \left(\frac{1}{\varepsilon} \int_{0}^{t} \sum_{s=0}^{m} \varepsilon^{s} \mu_{s}(\tau) d \tau\right)
$$

where

$$
\begin{gathered}
\mu_{2 k-2}(t)= \pm \mathrm{i} c_{2 k-2}(t), \quad \mu_{2 k-1}(t)=c_{2 k-1}(t) \quad(k \in \mathrm{N}) \\
c_{0}(t)=\sqrt{f_{3}(t) / f_{2}(t)} \\
c_{s}(t)=(-1)^{s}\left(f_{3}(t) \sum_{j=1}^{s-1} c_{j}(t) c_{s-j}(t)+f_{2}(t) c_{s-1}^{\prime}(t)+f_{1}(t) c_{s-1}(t)+\delta_{s, 2} f_{0}(t)\right) /\left(2 \sqrt{f_{2}(t) f_{3}(t)}\right)
\end{gathered}
$$

$\delta_{s, 2}$ is the Kronecker symbol.

Note that the functions $\mu_{s}(t)$ found by formulas (18), (20) - (22) are real-valued for an odd number $s$ and purely imaginary for an even $s$. Therefore, as an $m$-approximation to the general solution of differential equation (7), by virtue of formula (19), we choose the following real-valued function:

$$
\begin{gathered}
x(t ; \varepsilon)=\exp \left(\int_{0}^{t} \sum_{k=1}^{[(m+1) / 2]} \varepsilon^{2 k-2} c_{2 k-1}(\tau) d \tau\right) \\
\cdot\left\{D_{1} \cos \left(\frac{1}{\varepsilon} \int_{0}^{t} \sum_{k=0}^{[m / 2]} \varepsilon^{2 k} c_{2 k}(\tau) d \tau\right)+D_{2} \sin \left(\frac{1}{\varepsilon} \int_{0}^{t} \sum_{k=0}^{[m / 2]} \varepsilon^{2 k} c_{2 k}(\tau) d \tau\right)\right\},
\end{gathered}
$$

$[h]$ is the integer part of number $h$.

\section{Results}

In the formula (23), the numbers $D_{1}$ and $D_{2}$ so far are unknown, they must be determined from the boundary conditions (4) and the requirements that the solution (23) be nontrivial. Therefore, it is easy to see that the further analysis of the boundary value problem for eigenvalues (3) - (4) will depend on the boundary conditions specified in formula (4).

Further, let the boundary conditions (4) have the form:

$$
x(0)=x(T)=0 .
$$

Then, substituting expression (23) in the first of conditions (9), we obtain the value:

$$
D_{1}=0 \text {. }
$$


Therefore, as a solution to differential equation (3) under boundary conditions (24) with an error of order $\mathrm{O}\left(\varepsilon^{m}\right)$, the following function, obtained from (23) with value (25), can be taken:

$$
\begin{gathered}
\tilde{x}(t ; \varepsilon)= \\
D_{2} \sin \left(\int_{0}^{t} \sum_{k=0}^{[m / 2]} \varepsilon^{2 k-1} c_{2 k}(\tau) d \tau\right) \cdot \exp \left(\int_{0}^{t} \sum_{k=1}^{[(m+1) / 2]} \varepsilon^{2 k-2} c_{2 k-1}(\tau) d \tau\right) .
\end{gathered}
$$

Moreover, in the formula (26) should be $D_{2} \neq 0$.

We substitute expression (26) into the second of boundary conditions (24). This will result in the following equality:

$$
D_{2} \sin \left(\int_{0}^{T} \sum_{k=0}^{[m / 2]} \varepsilon^{2 k-1} c_{2 k}(\tau) d \tau\right) \cdot \exp \left(\int_{0}^{T} \sum_{k=1}^{[(m+1) / 2]} \varepsilon^{2 k-2} c_{2 k-1}(\tau) d \tau\right)=0 .
$$

Now taking into account the requirement of non-triviality of solution (26), i.e. the condition $D_{2} \neq 0$, from (27) we obtain the equation:

$$
\sin \left(\int_{0}^{T} \sum_{k=0}^{[m / 2]} \varepsilon^{2 k-1} c_{2 k}(\tau) d \tau\right)=0
$$

Therefore, from equation (28), returning from $\varepsilon$ to the initial parameter $\lambda$ by formula (6), we have the equality:

$$
\int_{0}^{T} \sum_{k=0}^{[m / 2]} \lambda^{1-2 k} c_{2 k}(\tau) d \tau=\pi n \quad(n \in \mathrm{N}) .
$$

Note that the value $n=0$ is excluded from formula (29), since it leads to the construction of only a trivial solution to the boundary value problem (3), (24). Negative integer values of $n$ can easily be reduced to case (29) described above due to the oddness of the function $\sin \mathrm{x}$.

Thus, equality (29) is the desired equation from which we find approximations of the required order $\mathrm{O}\left(\varepsilon^{m}\right)$ to the eigenvalues of the considered problem (3) with boundary conditions (24).

For example, in the first approximation (at $m=1$ ), the eigenvalues of the problem are determined by the formula:

$$
\lambda_{n}^{(1)}=\pi n / \int_{0}^{T} c_{0}(\tau) d \tau \quad(n \in \mathrm{N})
$$


If you choose $m=2$, then to find the second approximation $\lambda_{n}^{(2)}$ we obtain the following quadratic equation:

$$
z^{2} \int_{0}^{T} c_{0}(\tau) d \tau-z \pi n+\int_{0}^{T} c_{2}(\tau) d \tau=0
$$

Solving equation (31), we find:

$$
\lambda_{n}^{(2)}=\left(\pi n+\left((\pi n)^{2}-4 \int_{0}^{T} c_{0}(\tau) d \tau \cdot \int_{0}^{T} c_{2}(\tau) d \tau\right)^{1 / 2}\right) /\left(2 \int_{0}^{T} c_{0}(\tau) d \tau\right), \quad n \in \mathrm{N}
$$

It is easy to see that $\lambda_{n}^{(2)} \rightarrow \lambda_{n}^{(1)}$ with $n \rightarrow+\infty$, and $\lambda_{n}^{(2)}<\lambda_{n}^{(1)}$, if $\int_{0}^{T} c_{2}(\tau) d \tau>0$; and $\lambda_{n}^{(2)}>\lambda_{n}^{(1)}$ if $\int_{0}^{T} c_{2}(\tau) d \tau<0$. In addition, taking into account formulas (30) and (32), the equality is established:

$$
\begin{aligned}
\left|\lambda_{n}^{(2)}-\lambda_{n}^{(1)}\right|=\mid & \pi n-\left((\pi n)^{2}-4 \int_{0}^{T} c_{0}(\tau) d \tau \cdot \int_{0}^{T} c_{2}(\tau) d \tau\right)^{1 / 2} \mid / \\
& \left(2 \int_{0}^{T} c_{0}(\tau) d \tau\right), \quad n \in \mathrm{N} .
\end{aligned}
$$

By analogy, we can find asymptotic approximations $\lambda_{n}^{(m)}$ of any given order $m$ to the desired eigenvalues of the boundary value problem under study.

Moreover, by virtue of formula (26), the eigenfunctions corresponding to the number $\lambda_{n}^{(m)}$ of the boundary value problem under study (up to a constant) have an asymptotic representation:

$$
\begin{gathered}
x_{n}^{(m)}(t ; \varepsilon)= \\
\sin \left(\int_{0}^{t} \sum_{k=0}^{[m / 2]}\left(\lambda_{n}^{(m)}\right)^{1-2 k} c_{2 k}(\tau) d \tau\right) \cdot \exp \left(\int_{0}^{t} \sum_{k=1}^{[(m+1) / 2]}\left(\lambda_{n}^{(m)}\right)^{2-2 k} c_{2 k-1}(\tau) d \tau\right) .
\end{gathered}
$$

\section{Discussion of the results}

We demonstrate the computational capabilities of the applied use of the constructed asymptotics. Using the formulas (30) obtained above, we construct an asymptotic 
representation of the least order (first) of accuracy for the eigenvalues of the boundary value problem [15]:

$$
\left\{\begin{array}{l}
x^{\prime \prime}+\lambda^{2}\left(1-t^{2}\right) x=0 \\
x(0)=x(1)=0
\end{array}\right.
$$

System (35) is a special case of the problem (3), (24) studied above. In the case under consideration, in formulas (3) and (24) we have:

$$
f_{3}(t)=(1+t)^{2}, \quad f_{2}(t) \equiv 1, \quad f_{1}(t) \equiv 0, \quad f_{0}(t) \equiv 0, \quad T=1 .
$$

The fulfillment of conditions (5) for the boundary value problem (35) is verified directly.

To construct the first approximation (when $m=1$ ) to the solution of problem (35), it suffices to calculate the functions $c_{0}(t)$ and $c_{1}(t)$ by formulas (20) - (22). These functions, as shown above, satisfy the conditions:

$$
\int_{0}^{1} c_{0}(\tau) d \tau=3 / 2, \quad \int_{0}^{t} c_{1}(\tau) d \tau=-1 /(2 \ln (1+t)) .
$$

From formulas (37) we find:

$$
\begin{gathered}
\lambda_{n}^{(1)}=2 \pi n / 3 \quad(n \in \mathrm{N}) ; \\
x_{n}^{(1)}(t ; \varepsilon)=\left(\sin \left(\left(t^{2}+2 t\right) \cdot \pi n / 3\right)\right) / \sqrt{t+1}, \quad n \in \mathrm{N} .
\end{gathered}
$$

To analyze the computational capabilities of asymptotics (38), (39), we compare the efficiency of the asymptotic and numerical methods for solving the boundary value problem (35). First, for various values $n \in \mathrm{N}$, we calculate the eigenvalues of the boundary value problem (35) using the formula (38); secondly, we find a solution to problem (35) using numerical integration.

Comparison of the obtained results showed their good agreement. Since the asymptotic behavior of the eigenvalues and eigenfunctions (38) and (39) was constructed under the condition $n \rightarrow+\infty$, it was natural that the accuracy of the asymptotic formulas (38) and (39) will increase with increasing serial number $n$. However, it turned out that already for the first eigenvalue (when $n=1$ ) the error of the compared results is quite small, amounted to $1.6462 \%$.

With increasing number $n$, the accuracy of asymptotics (38) grows substantially and rapidly. For example, for the eighth eigenvalue (when $n=8$ in formula (38)), the error in the results obtained by the two methods is $0.0215 \%$, and for the tenth eigenvalue (when $n=10$ in formula (38)) it is only $0.0063 \%$.

For the boundary value problem (35), the corresponding eigenfunctions (up to a constant) are approximately calculated by formula (39) and show similar accuracy to numerical solutions.

We note that the advantage of the obtained asymptotics is not only that they give good agreement with exact solutions. One of the main advantages of asymptotic methods, in our opinion, is that they allow us to approximately construct functions that are of interest to us 
in an analytical form (as opposed to numerical methods), which is convenient for further studies and calculations.

\section{References}

1. R.J. Pacio, Th. Wetzel, Solar Energy 93 11-22 (2013) DOI: 10.1016/j.solener.2013.03.025

2. Y. Zhang, Y. Cai, et al., Solar Energy 164 47-57 DOI: 10.1016/j.solener. 2018.01.085

3. F.R. Gantmakher, Matrix calculus with applications in the theory of dynamical systems, 544 (URSS, Moscow, 2016)

4. E.A. Coddington, N. Levinson, Theory of Ordinary Differential Equations, 472 (Publishing House of LCI, Moscow, 2007)

5. N.B. Andersen, M. Flensted-Jensen, Bulletin of Russian universities. Maths 4, 127, 241-251 (2019) DOI: 10.20310/2686-9667-2019-24-124-241-251

6. S. Bauer, A. Smirnov, et al., Asymptotic methods in solid mechanics, 356 (Institute of Computer Research, Moscow, 2007)

7. D.B. Davletov, O.B. Davletov, et al., Herald of Omsk University 23, 3, 20-27 DOI: 10.25513/ 1812-3996.2018.23(3).20-27

8. A.M. Ilyin, A.R. Danilin, Asymptotic methods in analysis, 248 (Fizmatlit, Moscow, 2009)

9. R.P. Kuzmina, Asymptotic methods for ordinary differential equations, 328 (Institute for Computer Research, Moscow, 2015)

10. P.D. Miller, Applied Asymptotic Analysis, 467 (Providence: AMS Rhode Island, 2006)

11. V.V. Nemytsky, V.V. Stepanov, Qualitative theory of differential equations, 552 (URSS, Moscow, 2017)

12. G.S. Zhukova, Ukrainian Mathematical Journal 37, 1, 16-20 (1985) https://doi.org/10.1007/BF01056845

13. G.S. Zhukova, Ukrainian Mathematical Journal 40, 356-362 (1988) https://doi.org/10.1007/BF01057196

14. G.S. Zhukova, Nauchno-tekhnicheskiy vestnik Povolzh'ya 4, 15-18 (2019)

15. A. Naife, Introduction to perturbation methods: Per. from English, 535 (World, Moscow, 1984)

16. G.S. Zhukova, Sistemnyye tekhnologii 4 (33), 110-115 (2019) 